\title{
THE WO STARS AND THEIR ENVIRONMENT
}

\author{
V.F. POLCARO ${ }^{1}$, R. VIOTTI ${ }^{1}$, C. ROSSI ${ }^{2}$ and L. NORCI ${ }^{3}$ \\ ${ }^{1}$ Istituto di Astrofisica Spaziale, CNR, Via Enrico Fermi 21, 00044 Frascati, Italy \\ ${ }^{2}$ Istituto Astronomico, Università La Sapienza, Via Lancisi 29, 00161 Roma, Italy \\ ${ }^{3}$ Max-Plank Institut für Extraterrestrische Physik, Postfach 1603, \\ D-85740 Garching bei München, B.R. Deutschland
}

The WO stars form a subgroup of the WR class characterized by a strong O vi emission at $382 \mathrm{~nm}$ (Sanduleak 1971; Barlow \& Hummer 1982). In 1989-90 we observed intermediate resolution spectra of the four Sanduleak WO stars and of their surrounding nebulosities using the Bologna-Loiano $1.52 \mathrm{~m}$ and ESO-La Silla $1.52 \mathrm{~m}$ and $2.2 \mathrm{~m}$ telescopes (e.g., Polcaro et al. 1991, 1992,1994 in preparation). Fig. 1 shows that the spectra are similar but with some striking differences among the four stars. They can be arranged in a sequence in order of increasing ionization: Sand 2, Sand 1, Sand 5, Sand 4 (see Table 1). Sand 4 is a binary system, and the continuum of the 04 companion dominates over that of the WO star. In the other objects, the reddening-corrected continuum can be fitted by a $80000 \mathrm{~K} \mathrm{BB}$, in agreement with the high ionization level of their emission spectra.

Tab. 1 WO stars - emission-line equivalent widths

\begin{tabular}{llllll}
\hline line $(\AA)$ & ion & Sand 1 & Sand 2 & Sand 4 & Sand 5 \\
& & & & & \\
4686 & He II + C IV & - & 752 & 159 & 327 \\
5280 & O VI & 12 & 56 & - & 72 \\
5580 & O v & 26 & 160 & $>108$ & 215 \\
5820 & C IV & 139 & 2757 & 114 & 401 \\
6080 & O VIII & $?$ & p & 3 & 8 \\
6560 & He II + C IV & 24 & 88 & 104 & 102 \\
7060 & C IV & 17 & 98 & 97 & $74:$ \\
\hline
\end{tabular}

The structures and the chemical compositions of the surrounding nebulae appear to be different. Diffuse emission in the CiV $581 \mathrm{~nm}$ doublet has been observed in Sand 5 (Polcaro et al. 1991). Sand 4 is surrounded by an H II $468.6 \mathrm{~nm}$ emission region (Melnick \& Heydari-Malayeri 1991), embedded in a diffuse nebulosity (Dopita et al. 1990). We took five long-slit spectra, corresponding to the central star and to a number of structures identified on the $\mathrm{H} \alpha+[\mathrm{N} \mathrm{II}]$ images by Dopita et al. (1990). We found that the $\mathrm{H} \alpha,[\mathrm{N} \mathrm{II}]$, [S II] and [Ar III] lines are strongly variable depending on the postion in the 

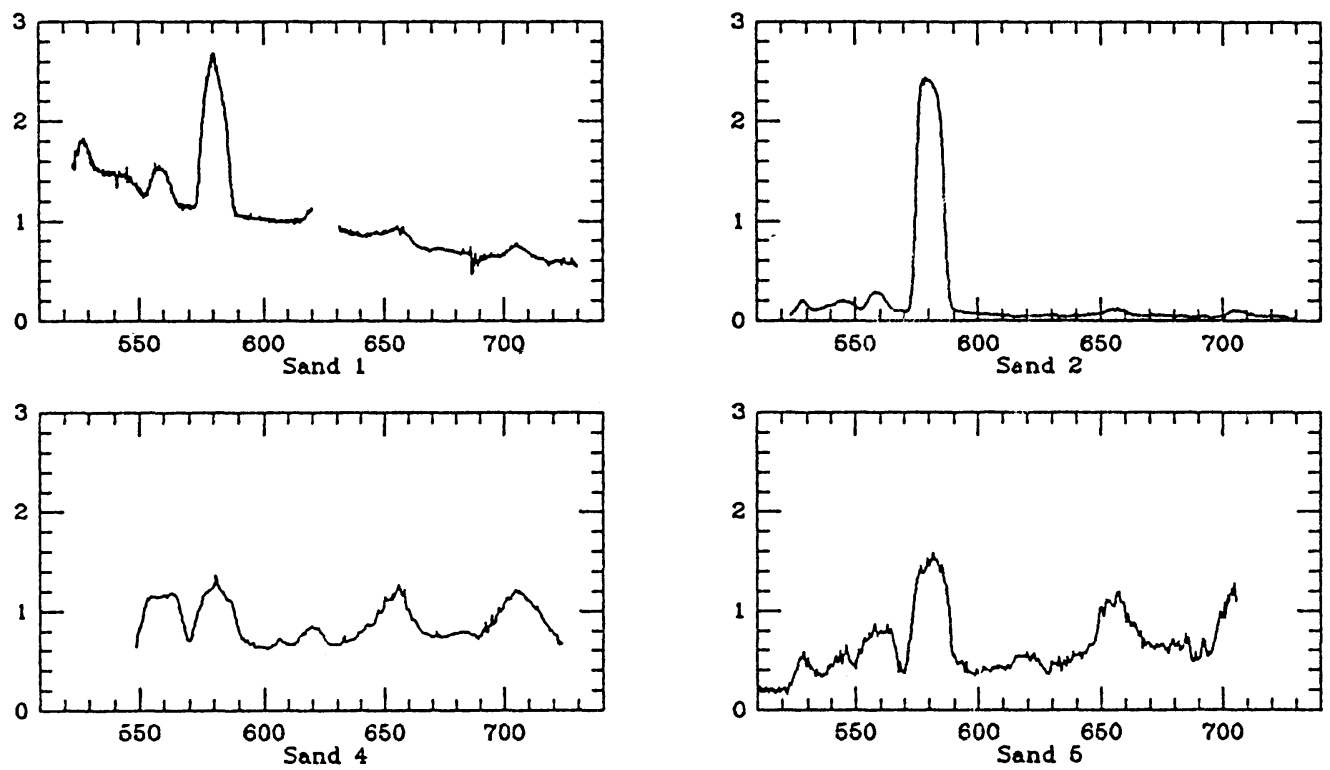

Fig. 1. The optical spectra of the WO stars

nebula. We also noted that the [N II] pattern is anomalous with respect to the other lines. He II $468.6 \mathrm{~nm}$ line is present not only around the central star but also in some of the northern structures. Long slit spectra of the LMC star Sand 2 revealed an asymmetric nebulosity clearly connected to the central object, visible at least in $\mathrm{H} \alpha$ and [N II] wavelengths.

\section{References}

Barlow, M.J., Hummer, D.C. 1982, in: C.W.H. de Loore \& A.J. Willis (eds.), Wolf-Rayet

Stars: Observations, Physics, Evolution, Proc. IAU Symp. No. 99 (Dordrecht: Reidel), p. 387

Dopita, M.A., Lozinskaya, T., McGregor, P.J., Rawling, S.J. 1990, ApJ 351, 563

Melnick, J., Hyedari-Malayeri, M. 1991, in: K.A. van der Hucht \& B. Hidayat (eds.), Wolf-

Rayet Stars and Interrelations with Other Massive Stars in Galaxies, Proc. IAU Symp.

No. 143 (Dordrecht: Kluwer), p. 409

Polcaro, V.F., Rossi, C., Norci, L., Giovannelli, F., Manchanda, R.K. 1991, $A \& A$ 252, 590

Polcaro, V.F., Viotti, R., Rossi, C., Norci, L. 1992, A\&A 265, 563

Sanduleak, N. 1971, ApJ (Letters) 164, L71 